
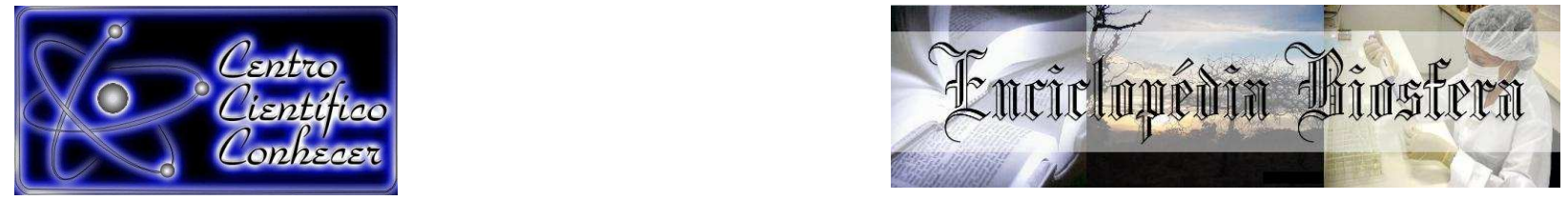

\title{
LESÃO RENAL TUBULAR AGUDA EM CÃES E GATOS: FISIOPATOGENIA E DIAGNÓSTICO ULTRASSONOGRÁFICO
}

Nathália Bragato1, Maria Clorinda Soares Fioravanti², Larissa Graciano Braga ${ }^{3}$, Danielly Cunha dos Reis ${ }^{3}$, Naida Cristina Borges ${ }^{2}$

${ }^{1}$ Mestranda, Escola de Medicina Veterinária e Zootecnia da Universidade Federal de Goiás, Goiânia, Brasil, email: nathaliabragato@yahoo.com.br

${ }^{2}$ Doutora, Docente da Escola de Medicina Veterinária e Zootecnia da Universidade Federal de Goiás, Goiânia, Brasil

${ }^{3}$ Graduanda, Escola de Medicina Veterinária e Zootecnia da Universidade Federal de Goiás, Goiânia, Brasil

Recebido em: 08/09/2015 - Aprovado em: 14/11/2015 - Publicado em: 01/12/2015 DOI: http://dx.doi.org/10.18677/Enciclopedia_Biosfera_2015_183

\begin{abstract}
A lesão renal aguda resulta principalmente da ação de agentes nefotóicos e de isquemia renal, que geralmente afetam a porção tubular do néfron. A detecção precoce da lesão renal aguda permite a intervenção apropriada podendo prevenir ou atenuar o dano à célula tubular e o desenvolvimento da insuficiência renal aguda. $O$ diagnóstico requer a associação de técnicas, sendo eficaz o emprego do exame ultrassonográfico associado aos exames laboratoriais. A ultrassonografia permite a avaliação da morfologia e da ecogenicidade renal por meio do exame em modo bidimensional e da hemodinâmica renal por meio da avaliação com o Doppler pulsado pelo cálculo dos índices de resistividade e pulsabilidade. As principais alterações ultrassonográficas da lesão tubular aguda são nefromegalia, aumento ou redução da ecogenicidade cortical, dilatação da pelve renal, efusão perirrenal, sinal de margem medular e aumento dos índices de resistividade e pulsabilidade.
\end{abstract}

PALAVRAS-CHAVE: ecografia, índice de pulsabilidade, índice de resistividade, lesão renal aguda

\section{ACUTE TUBULAR KIDNEY INJURY IN DOGS AND CATS: PHYSIOPATHOGENY AND ULTRASOUND DIAGNOSIS}

\begin{abstract}
Acute kidney injury resulting from toxic or ischemic insults and usually affect the tubular portion of the nephron. Early detection allows the acute kidney injury appropriate intervention may prevent or mitigate damage to the tubular cell and the development of acute renal failure. Diagnosis requires a combination of techniques, and be effective employment ultrasonography associated with laboratory tests. Ultrasound allows assessment of morphology and renal echogenicity by taking on bidimensional mode and renal hemodynamics through the assessment with pulsed Doppler with the calculation of resistivity and pulsatility indices. The main ultrasound changes of acute tubular injury are nefromegalia, cortical echogenicity increase or
\end{abstract}


reduction, renal pelvis expansion, perirenal effusion, medular signal margin and resistivity and pulsatility indices increase.

KEYWORDS: acute kidney injury, echography, pulsatility index, resistivity index

\section{INTRODUÇÃO}

Para garantir a adequada filtração do plasma, os rins recebem aproximadamente $25 \%$ do débito cardíaco e possuem uma alta taxa metabólica, principalmente na região dos túbulos renais, devido a sua função de reabsorver substâncias filtradas e secretar metabólitos e substâncias exógenas que devem ser eliminadas (GRAUER, 2005; LUNN, 2011). Com isso as células tubulares ficam expostas a altas concentrações de agentes potencialmente nefrotóxicos e se tornam mais susceptíveis a danos provocados por isquemia, que provocam necrose tubular aguda (NTA) e podem evoluir para insuficiência renal aguda (IRA).

A ocorrência de IRA em cães e gatos tem sido relatada principalmente em ambiente hospitalar devido ao tratamento com fármacos nefrotóxicas, uso de medicamentos que reduzem o fluxo sanguíneo renal, de diuréticos e hipoperfusão renal em cirurgias e anestesias prolongadas (LUNN, 2011). No entanto, na maioria dos casos há um ou mais fatores predisponentes associados como idade avançada, doença renal pré-existente, insuficiência cardíaca e desidratação, que podem agravar a lesão renal (GRAUER, 2005; RUFATO et al., 2011).

Cães e gatos com lesão renal aguda apresentam um prognóstico bastante variável, que depende principalmente do diagnóstico e do tratamento precoce, pois a manutenção da membrana basal é essencial para a regeneração das células tubulares e recuperação do néfron (GRAUER, 2005; LUNN, 2011; ROSS, 2011). Na maioria dos casos o diagnóstico é tardio, quando o animal já apresenta IRA, o que faz com que a taxa de mortalidade e o número de pacientes que evoluem para doença renal crônica sejam muito elevados, resultando em um prognóstico desfavorável (VADEN et al., 1997; DIEHL \& SESHADRI, 2008; DORVAL \& BOYSEN, 2009; COOPER \& LABATO, 2011).

Portanto, o diagnóstico precoce é fundamental para se estabelecer o prognóstico e evitar a progressão para IRA, sendo indispensáveis os exames clínico, laboratoriais e ultrassonográfico para a avaliação da função e da integridade renal. $A$ ultrassonografia, tanto em modo bidimensional (modo B) quanto em modo Doppler, tem se mostrado como uma importante ferramenta de diagnóstico da lesão tubular aguda, pois permite a visualização de alterações que podem ocorrer na morfologia, na ecogenicidade e na hemodinâmica renal, além de auxiliar no acompanhamento e determinação do prognóstico juntamente com os resultados dos exames laboratoriais.

Com esta revisão de literatura objetivou-se descrever a fisiopatogenia, a etiologia e as alterações ultrassonográficas em modo B e Doppler pulsado que ocorrem na lesão renal tubular aguda em cães e gatos.

\section{FISIOPATOGENIA}

A lesão renal tubular aguda possui uma fisiopatogenia complexa que está relacionada principalmente a isquemia à ação direta de agentes nefrotóxicos nas células tubulares (LUNN, 2011). Nos casos de isquemia a bomba ATPase $\mathrm{Na}+\mathrm{K}+$ tem seu funcionamento alterado devido a falta de ATP, o que faz com que o cálcio entre nas células tubulares provocando apoptose e NTA (JOHN \& HERZEMBERG, 2009; MENEZES et al., 2010; ROSS, 2011). No caso dos agentes nefrotóxicos, 
estes são para o interior do túbulo para serem excretados, mas se presentes em grande quantidade acumulam-se nos lisossomos, interferindo na ação das fosfolipases sobre o catabolismo dos fosfolipídios, resultando em fosfolipidose lisossomal que leva a ruptura dos lisossomos e necrose das células epiteliais tubulares proximais (MENEZES et al., 2010), os agentes nefrotóxicos também inibem a função das mitocôndrias e estimulam a produção de radicais livres agravando o dano às células tubulares (JOHN \& HERZEMBERG, 2009).

Durante o processo de filtração renal os túbulos contornados proximais absorvem grande quantidade de água e eletrólitos, a necrose deste tecido leva a poliúria devido a redução da reabsorção tubular. $O$ excesso de sódio e água nos túbulos faz com que estes extravasem para o espaço intersticial provocando edema e a falta de ATP provoca ruptura do citoesqueleto e descamação das células tubulares, estes dois fatores contribuem para a obstrução tubular levando a oligúria ou anúria. As proteínas de baixo peso molecular que conseguem ultrapassar a barreira glomerular deixam de ser reabsorvidas pelos túbulos lesados resultando em proteinúria (LUNN, 2011; ROSS, 2011).

A obstrução tubular, o extravasamento tubular retrógrado devido ao excesso de água e solutos nos túbulos e a vasoconstrição renal provocada pela reação inflamatória, levam a redução da taxa de filtração glomerular e perda da função do néfron e, caso uma área renal muito extensa seja afetada, o animal se torna portador de IRA (COSTA et al.; 2003; ROSS, 2009). A IRA é definida como redução ou perda súbita da função renal, resultando em inabilidade em excretar compostos nitrogenados (MELCHERT et al., 2007; ROSS, 2011), acompanhada ou não da diminuição da diurese e perda da capacidade de concentrar a urina (COSTA et al., 2003).

A IRA ocorre dentro de horas ou dias após a agressão renal, prejudicando a regulação do equilíbrio hidroeletrolítico e ácido base (GRAUER, 2005; MYOTT \& LANGSTON, 2011). Suas principais complicações são a perda excessiva de água e sódio levando a desidratação nos casos de poliúra (ROSS, 2009), falha dos túbulos de reabsorver bicarbonato e secretar ácido levando a acidose metabólica, falha em excretar o excesso de potássio do organismo promovendo hipercalemia, acúmulo de toxinas urêmicas na circulação resultando em azotemia (BERBEL et al., 2011) e retenção de água e sódio nos casos de oligúria ou anúria que resultam em hipertensão arterial (ACIERMO \& LABATO, 2005).

O dano celular ocorre em cascata e, se o estímulo causador não for removido, a própria ação da NTA promove vasoconstrição e isquemia renal agravando a lesão, o que pode afetar a membrana basal fazendo com que o túbulo não consiga se regenerar (COSTA et al., 2003; LUNN, 2011). Mesmo que a recuperação da função renal seja incompleta, o funcionamento renal adequado pode ser restabelecido devido à hipertrofia compensatória morfológica e funcional dos néfrons que não foram irreversivelmente lesados.

Entretanto, as agressões renais agudas, caracterizadas pelos quadros de IRA, independente de sua causa, podem resultar em substituição do parênquima renal por tecido cicatricial, reduzindo a massa renal funcional. Dependendo da extensão da perda, o paciente pode tornar-se portador de doença renal crônica, que pode evoluir para insuficiência renal crônica nos casos mais graves (POLZIN, 2011; VEADO, 2011). 


\section{ETIOLOGIA}

Várias são as causas de lesão renal aguda, elas podem ser classificadas em pré-renais, renais intrínsecas e pós-renais (FRANCEY \& SCHWEIGHAUSER, 2009). As causas pré-renais provocam redução da perfusão renal levando a isquemia, portanto sendo denominada lesão renal isquêmica. Inicialmente não há alteração estrutural nos rins e o diagnóstico precoce é extremamente importante, pois se a perfusão renal for reestabelecida dentro de um a dois dias o quadro será reversível, caso contrário os danos isquêmicos provocam NTA e consequentemente a IRA (COSTA et al., 2003). Os principais fatores que levam a isquemia renal são o uso indiscriminado de anti-inflamatórios não esteroides (AINEs) que causam a redução da produção de prostaglandinas renais levando a vasoconstrição e hipóxia renal (MELGAÇO et al., 2010; KHAN \& MCLEAN, 2012), o uso de diuréticos e de fármacos hipotensores como os inibidores da enzima conversora da angiotensina (ECA) (FRANCEY \& SCHWEUGHAUSER, 2009; LUNN, 2011), hipovolemia, fluidoterapia e oxigenoterapia inadequadas durante a anestesia ou procedimentos cirúrgicos prolongados provocando hipoperfusão renal (BAETGE \& MATTHEWS, 2012).

As causas renais intrínsecas ocorrem devido a ação de nefrotoxinas (MELCHERT et al., 2007), doenças infecciosas parenquimais como pielonefrite (FRANCEY \& SCHWEIGHAUSER, 2009) e leptospirose (LANGSTON \& HEUTER, 2009) e glomerulonefrite devido a deposição de complexos antígeno-anticorpo nas hemoparasitoses e na leishmaniose (LITTMAN, 2011).

O uso de fármacos potencialmente nefrotóxicos é uma das principais causas de lesão tubular, sendo os mais comuns os aminoglicosídeos, o paracetamol, a anfotericina B (MELCHERT et al., 2007), a cisplatina e os agentes de contraste radiográfico iodados (GRAUER, 2005). As lesões por fármacos nefrotóxicos estão relacionadas principalmente a sua concentração, a duração de exposição e a múltiplos fatores e resistência do paciente, portanto seu uso deve ser restrito. Estes medicamentos raramente causam lesão tubular em pacientes jovens e sadios, no entanto podem representar maior risco para pacientes idosos, desidratados ou que possuem alguma nefropatia subclínica (JOHN \& HERZEMBERG, 2009). Geralmente, a lesão nefrotóxica é reversível e passível de correção se identificada e corrigida precocemente (COSTA et al., 2003).

As causas pós-renais são representadas por obstrução do fluxo urinário, que levam a retenção de urina na pelve e perda da função renal por compressão do parênquima (MELCHERT et al., 2007). Estudo realizado por COSTA et al. (2003) demonstrou que as principais causas de lesão renal aguda em seres humanos foram isquemia em $50 \%$ dos casos, devido a perdas excessivas de fluídos provocados por doenças gastrointestinais, inadequada reposição de fluidos, insuficiência cardíaca e o uso de diuréticos; toxinas em $35 \%$ dos casos; nefrite intersticial em $10 \%$ dos casos e glomerulonefrite aguda em $5 \%$ dos casos. LUNN (2011) descreveu que as principais causas de lesão tubular aguda em cães são isquemia e exposição a agentes nefrotóxicos, ou possuem causa desconhecida e, em gatos a principal causa é o uso de agentes nefrotóxicos.

Em estudo realizado por VADEN et al. (1997), que revisaram os registros médicos de cães e gatos de um hospital veterinário, em 99 casos de IRA, as causas mais comuns foram isquemia, seguidas por exposição a nefrotóxicos. Neste estudo a maioria dos animais possuíam múltiplas desordens associadas, condizendo com a natureza multifatorial da IRA. COOPER \& LABATO (2011) em estudo com 22 gatos com IRA observaram que as principais causas foram obstrução urinária em $36 \%$ dos 
casos, descompensação de doença renal crônica em $32 \%$ dos casos, nefrotoxinas em $18 \%$ dos casos e a causa foi desconhecida em $14 \%$ dos casos.

\section{FATORES DE RISCO}

Existem vários fatores de risco relacionados à lesão renal tubular aguda, sendo estes fatores aditivos quando combinados e qualquer complicação em pacientes de risco aumenta o potencial para o desenvolvimento de IRA (GRAUER, 2005; RUFATO et al., 2011). Idade avançada, doença renal pré-existente (KUKANICH, 2012), insuficiência cardíaca congestiva (ROSS, 2012), pancreatite (LI et al., 2010), coagulação intravascular disseminada, acidose, choque, sepse, febre, causas que podem levar a hipotensão, como desidratação, trauma, queimaduras extensas, anestesias prolongadas, cirurgias, o uso de inibidores da ECA, AINEs e de fármacos potencialmente nefrotóxicos são os principais fatores de risco relacionados ao desenvolvimento de IRA (COSTA et al., 2003; GRAUER, 2005). Cães com piometra também possuem alto risco de desenvolver IRA, pois a endotoxina da Escherichia coli induz a redução da capacidade de concentrar a urina, principalmente quando a reposição de fluidos é inadequada durante a ovarioesterectomia e no período de recuperação (GRAUER, 2005; SANTOS et al., 2013; ANDRADE et al., 2014).

Em muitos casos, a IRA ocorre no ambiente hospitalar durante a realização de procedimentos diagnósticos ou terapêuticos, sendo um problema significante na medicina e na medicina veterinária, principalmente nas unidades de terapia intensiva (UTI) (COSTA, 2003; LUNN, 2011), onde, em seres humanos, a incidência pode chegar a $23 \%$ dos pacientes (COSTA et al., 2003). Em humanos a IRA adquirida em ambiente hospitalar geralmente resulta de múltiplos fatores como hipovolemia, sepse, medicamentos nefrotóxicos, cirurgias prolongadas e baixo débito cardíaco (COSTA et al., 2003). Em cães, as principais causas são drogas nefrotóxicas, como aminoglicosídeos e cisplatina, o uso de AINEs, inibidores da ECA, doença cardíaca crônica e doenças renais pré-existentes. Cães com idade superior a sete anos apresentam maior risco de desenvolver IRA no ambiente hospitalar e maior taxa de mortalidade (LUNN, 2011).

\section{PROGNÓSTICO}

O prognóstico para cães e gatos que desenvolvem IRA tem se mostrado fortemente dependente da causa e da extensão da lesão renal, além da idade do paciente e da presença de doença renal pré-existente ou falha orgânica (ROSS, 2009). O tratamento é dispendioso e requer vários dias de hospitalização até o retorno da função renal, que, se ocorrer, pode levar meses (MELCHERT et al., 2007) e requerer o uso de terapia de substituição renal por tempo indeterminado (INTERNATIONAL RENAL INTEREST SOCIETY, 2013).

As lesões tubulares agudas são responsáveis pelo desencadeamento de reações inflamatórias, que podem terminar com a formação de áreas de cicatrização e, consequentemente, perda da massa renal funcional (VEADO, 2011). Se esta perda for extensa, caracterizada por diminuição de tecido de dois terços a três quartos de ambos os rins, surgem os sinais permanentes de incapacidade de execução de uma ou mais funções renais e, esses pacientes, evoluem para insuficiência renal crônica (POLZIN, 2011).

Estudos têm indicado prognóstico desfavorável associado à IRA. Em humanos, a taxa de mortalidade é alta (50\% a $60 \%)$, apesar da disponibilidade de 
diálise. A morte desses pacientes é frequentemente o resultado de lesões em órgãos distantes, secundárias à IRA e/ou uremia (COSTA, 2003).

Resultados de vários estudos clínicos, realizados a partir de 2008, indicaram elevadas taxas de mortalidade em cães e gatos, semelhante às observadas em humanos. DORVAL \& BOYSEN (2009) avaliaram seis gatos com IRA, tratados com diálise peritoneal, cinco vieram a óbito e apenas um sobreviveu. COOPER \& LABATO (2011) avaliaram 22 gatos com IRA, também tratados com diálise peritoneal, e observaram taxa de mortalidade de 54,5\%. DIEHL \& SESHADRI (2008) avaliaram 17 cães e 16 gatos com IRA tratados com hemodiálise e foi observada taxa de mortalidade de $35 \%$ nos cães e $56 \%$ nos gatos.

A taxa de mortalidade de cães e gatos com IRA foi de 56\% em estudo realizado por VADEN et al. em 1997, sendo esta taxa semelhante à encontrada nos estudos mais recentes, portanto, mesmo com os avanços no tratamento nos últimos anos o prognóstico para IRA ainda permanece desfavorável.

Casos de lesão pós-renal devido à obstrução do fluxo urinário, quando tratados antes do desenvolvimento de hidronefrose e destruição do parênquima renal, indicam prognóstico mais favorável quando comparado à lesão renal intrínseca (ROSS, 2009). Casos de IRA tóxica apresentam melhor prognóstico em relação aos de IRA isquêmica, pois geralmente nos casos de nefrotoxicidade a membrana basal das células tubulares é preservada, o que faz com que, cessado o estímulo tóxico, ocorra regeneração e proliferação do epitélio tubular (GRAUER, 2005). Na IRA isquêmica frequentemente é observada atrofia tubular e fibrose intersticial intensas que atingem a membrana basal, impossibilitando a regeneração do epitélio tubular (MENEZES et al., 2010).

De acordo com VADEN et al. (1997) pior prognóstico e falha em recuperar a função renal estão relacionados a ocorrência de azotemia, hipocalemia, anemia, proteinúria, doença renal crônica e falha em converter anúria ou oligúria para estado não oligúrico. COLE et al. (2012) e ROSS, (2012) relatam que a insuficiência cardíaca aguda descompensada também é um fator que define um prognóstico desfavorável na IRA

O diagnóstico precoce e a identificação da causa da lesão tubular aguda aumentam a taxa de sobrevivência dos pacientes, pois permitem a intervenção apropriada podendo prevenir ou atenuar o dano à célula tubular, minimizando 0 a ocorrência de lesão renal persistente que leva ao desenvolvimento da IRA (GRAUER, 2005; LUNN, 2011; PALUMBO et al., 2011; ROSS, 2011) ou a progressão para doença renal crônica (VEADO et al, 2011).

A Sociedade Internacional de Interesse Renal (International Renal Interest Society - IRIS) desenvolveu uma classificação em cinco graus da injúria renal aguda para a medicina veterinária, a fim de proporcionar uma ferramenta para seu reconhecimento precoce, padronizar o grau de lesão renal, o curso clínico e o prognóstico. No grau 1 são incluídos pacientes não azotêmicos (creatinina menor que $1,6 \mathrm{mg} / \mathrm{dl}$ ) com presença de injúria renal aguda documentada por evidência de alteração renal nos exames de imagem, presença de oligúria ou anúria, ou aumento progressivo da creatinina igual ou superior a $0,3 \mathrm{mg} / \mathrm{dl}$ em intervalo de 48 horas. No grau 2 incluem pacientes com azotemia estática ou progressiva, com creatinina entre 1,7 e 2,5mg/dl. Os graus 3, 4 e 5 incluem pacientes com azotemia moderada a severa, com valores de creatinina entre 2,6 a 5,0mg/dl, 5,1 a 10,0mg/dl e acima de $10 \mathrm{mg} / \mathrm{dl}$, respectivamente (INTERNATIONAL RENAL INTEREST SOCIETY, 2013).

Animais com classificação IRIS grau 1 e 2 possuem melhor prognóstico, podendo recuperar a função renal adequada dentro de dois a três dias e geralmente 
requerem suporte a curto prazo. Pacientes com graus IRIS maiores, cujo grau progride durante a internação, podem exigir semanas de cuidado de suporte para a recuperação renal. Animais com IRA grave, com classificação IRIS graus 4 e 5 podem morrer dentro de cinco a dez dias apesar do manejo convencional adequado, a menos que tratados com terapia de substituição renal por tempo indeterminado. Essa disparidade entre a janela de sobrevivência com a terapia de suporte convencional e o tempo prolongado necessário para reparar a injúria renal grave, fundamenta o mal prognóstico e resultados associados aos estágios graves da IRA (INTERNATIONAL RENAL INTEREST SOCIETY, 2013).

\section{DIAGNÓSTICO}

A lesão renal pode ser detectada por testes laboratoriais, exames de imagem e avaliação histopatológica do tecido renal. A distinção entre insuficiência renal reversível e irreversível fundamentada em achados clínicos é difícil de ser realizada, uma vez que os sinais não são específicos o bastante para 0 estabelecimento do diagnóstico definitivo. A maioria dos testes de função renal indica a competência funcional dos rins no momento em que são realizados, não possibilitando a determinação da duração, do tipo de lesão ou da evolução do quadro (LUNN, 2011).

Pacientes com uremia aguda apresentam diversos sinais clínicos devido à ação das toxinas urêmicas. Os principais sinais são náuseas, vômito e presença de úlceras gástricas, que levam a anorexia. Anemia pode ocorrer em decorrência da fragilidade eritrocitária com redução da meia vida das hemácias pela ação das toxinas, em casos mais graves a anemia também resulta da diminuição da produção de eritropoietina e perda sanguínea devido à presença de úlceras no trato gastrointestinal, o sangramento pode ser potencializado pela disfunção plaquetária urêmica (ROSS, 2009). A uremia aguda também provoca um elevado catabolismo no organismo, que é agravado pela redução da excreção renal de ácido e da reabsorção de bicarbonato que resulta em acidose metabólica. Estes dois fatores exacerbam a azotemia, a hipercalemia, a hiperfosfatemia e a perda de massa magra corporal, agravando o quadro clínico (ROSS, 2009; BERBEK et al., 2011).

A produção de urina varia de modo significativo entre os pacientes com lesão renal aguda, podendo ocorrer poliúria, oligúria ou anúria (ROSS, 2009). Alterações na produção de urina, associadas à alterações no estado metabólico e no balanço hidroeletrolítico, podem levar ao colapso circulatório e danos em outros órgãos e sistemas devido a desidratação, nos casos em que ocorrem poliúria, ou hipervolemia e hipertensão, nos casos de oligúria ou anúria (DIEHL \& SESHADRI, 2008).

Estudo realizado por ACIERMO \& LABATO (2005) demonstra que a hipertensão está presente em $50 \%$ a $93 \%$ dos cães e $19,4 \%$ a $61 \%$ dos gatos com doença renal. Segundo estes autores, a elevação da pressão arterial nos casos de lesão renal aguda está relacionada principalmente à expansão aguda de volume secundária a oligúria com retenção de sódio e água e, à vasoconstrição secundária a produção de renina.

Os principais achados laboratoriais da lesão renal aguda são azotemia, enzimúria, cilindrúria, proteinúria, glicosúria, descamação de células renais e piúria (GRAUER, 2005). Em estudo realizado por MELCHERT et al. (2007) com indução de lesão renal aguda por gentamicina, o achado laboratorial indicativo de lesão tubular mais precoce foi o aumento da enzima gama glutamiltransferase na urina, que ocorreu no terceiro dia de intoxicação. A cilindrúria também ocorreu 
precocemente, no quarto dia, com aumento da intensidade no sétimo dia, porém esta alteração não foi observada em todos os animais. Aumento das concentrações de ureia e creatinina ocorreram apenas no sétimo dia de intoxicação, demonstrando que estes são bons indicadores para avaliação da TFG, mas não para a determinação de lesão tubular de forma precoce (MELCHERT et al., 2007).

A ultrassonografia em modo B é o exame de escolha para a avaliação da morfologia renal, pois permite a visibilização da arquitetura e dimensões renais (NYLAND \& MATTOON, 2005). A ultrassonografia Doppler é empregada de forma complementar ao exame em modo-B (CARVALHO et al., 2009), uma vez que alterações da perfusão, em muitos casos, podem ser os primeiros sinais de que a funcionalidade renal está alterada (MELO et al., 2006).

\section{ULTRASSONOGRAFIA EM MODO B}

A lesão tubular aguda provoca alteração renal difusa que pode ser de difícil identificação, ou pode não provocar nenhuma alteração na ultrassonografia convencional em modo B (NYLAND \& MATTOON, 2005). A causa não pode ser definida apenas pela ultrassonografia, e, quando não puder ser definida clinicamente, o exame histopatológico renal poderá contribuir um resultado confirmatório (CARVALHO et al., 2010; PRADO et al., 2014).

Uma das principais alterações ultrassonográficas que pode ser vista na lesão tubular aguda é a nefromegalia, que resulta de diferentes processos fisiopatológicos como o edema, infiltração inflamatória e hipertrofia compensatória (HOLLOWAY \& O'BRIEN, 2007; LANGSTON \& HEYTER, 2009; ROSS, 2011). O grau do aumento associa-se à severidade da lesão renal e, geralmente, é bilateral (MONTEIRO \& FROES, 2009).

A mensuração renal pode ser obtida pela ultrassonografia com alto grau de precisão, refletindo as verdadeiras dimensões dos rins, analisados à necropsia (BARR et al., 1990). No entanto não existe um parâmetro de referência para o volume renal de cães saudáveis devido à ampla variação racial e amplitude de peso corporal (SAMPAIO \& ARAÚJO, 2002; CUNHA, 2005).

Estudos têm mostrado alta correlação entre o peso corporal e o volume renal, tendo sido descritos a média do volume renal por quilo em cães saudáveis: 2,21 a 2,8 $\mathrm{cm}^{3} / \mathrm{kg}$ (BARR et al., 1990), 1,99 a 2,30 $\mathrm{cm}^{3} / \mathrm{kg}$ (SAMPAIO \& ARAÚJO, 2002), e 1,0 a $2,20 \mathrm{~cm}^{3} / \mathrm{kg}$ para o rim direito e 1,27 a $2,55 \mathrm{~cm}^{3} / \mathrm{kg}$ para o rim esquerdo (CUNHA, 2005). Porém os valores variam muito e possuem alto desvio padrão o que limita sua aplicação para detectar aumentos discretos do volume renal (NYLAND \& MATTOON, 2005), assim, a avaliação seriada ou o conhecimento do tamanho renal do cão enquanto saudável é importante para detectar aumentos discretos do volume renal.

Alterações de ecogenicidade são frequentes na lesão tubular aguda, podendo ocorrer tanto aumento como redução da ecogenicidade cortical. Uma análise comparativa com os outros órgãos abdominais normalmente é realizada para determinar a ecogenicidade do córtex renal, sendo esta comparada com a ecogenicidade do fígado e do baço (SANTOS et al., 2013). O rim esquerdo deve possuir córtex hipoecoica quando comparada ao baço, enquanto no rim direito a córtex deve ser isoecogênica ou ligeiramente hipoecoica em relação ao parênquima hepático, quando comparados na mesma profundidade (Figura 1) (NYLAND \& MATTOON, 2005). 

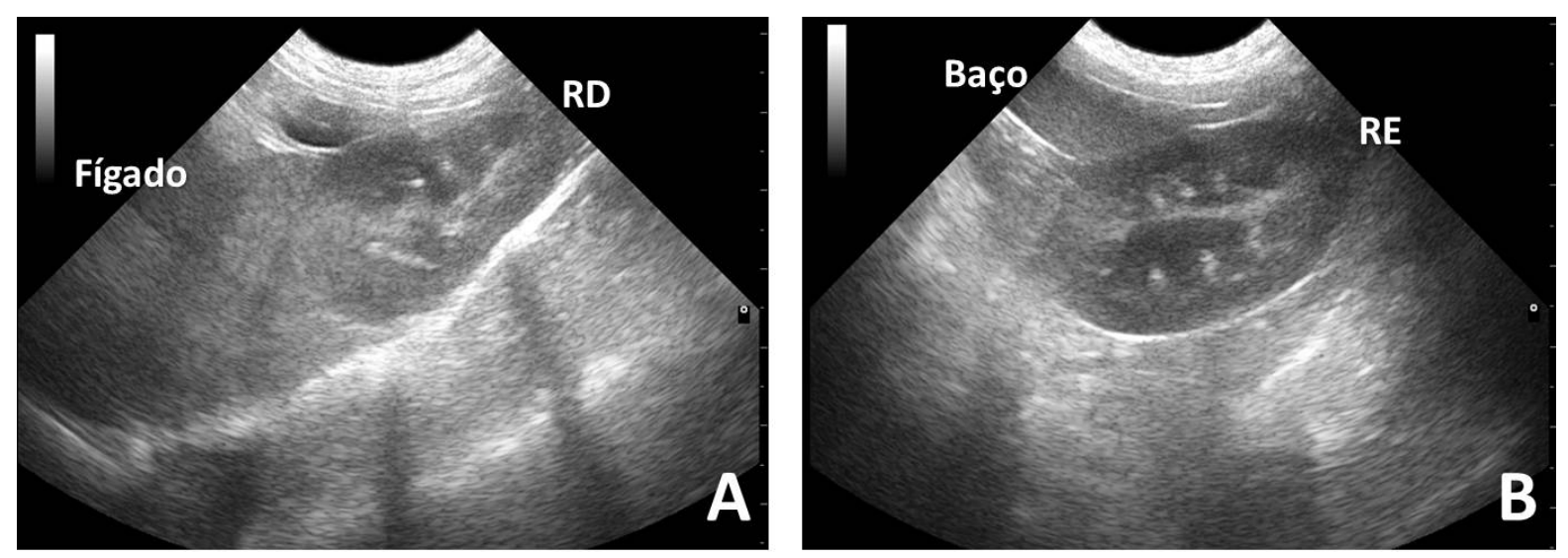

FIGURA 1 - Ultrassonografia abdominal de cão adulto saudável mostrando a relação de ecogenicidade normal entre baço, fígado e rim. A - córtex do rim direito $(R D)$ isoecoico em relação ao fígado. $B$ - córtex do rim esquerdo (RE) hipoecoico em relação ao baço

Uma mudança acentuada nas relações de ecogenicidade entre baço, fígado e rim sugere a possibilidade de alterações patológicas em um ou mais desses órgãos. Porém a ecogenicidade de um órgão pode variar de acordo com a frequência e o tipo de transdutor utilizado e da profundidade da imagem no equipamento (NYLAND \& MATTOON, 2005).

Aumento da ecogenicidade cortical renal pode ser facilmente percebido, pois, comparando-se com a ecogenicidade do baço e do fígado, esta estará aumentada em relação a esses órgãos (Figura 2) (NYLAND \& MATTOON, 2005). $O$ aumento de ecogenicidade pode ocorrer devido à fibrose ou esclerose nas doenças renais crônicas, ou por infiltrado celular e presença de cilindros celulares ou proteináceos e debris celulares nos túbulos renais em casos de nefrite glomerular e intersticial aguda, necrose ou nefrose tubular aguda (NYLAND \& MATTOON, 2005; HOLLOWAY \& O'BRIEN, 2007; LANGSTON \& HEUTER, 2009; CARVALHO et al., 2010; ROSS, 2011).

ARAÚJO et al. (2010) encontraram correlação entre o aumento de ecogenicidade cortical e anormalidades túbulo intersticiais vistas no exame histopatológico em humanos, além de alta correlação entre o grau de ecogenicidade e a gravidade dos achados histológicos e o aumento da creatinina. Demonstrando que a ecogenicidade cortical é útil para o diagnóstico da lesão tubular aguda e seu acompanhamento.

A redução da ecogenicidade cortical ocorre resultante de edema associado à inflamação aguda (NYLAND \& MATTOON, 2005; HOLLOWAY \& O'BRIEN, 2007 LANGSTON \& HEUTER, 2009; ROSS, 2011), porém esta é mais difícil de ser detectada, pois não há um parâmetro de comparação com outros órgãos como no aumento da ecogenicidade. 

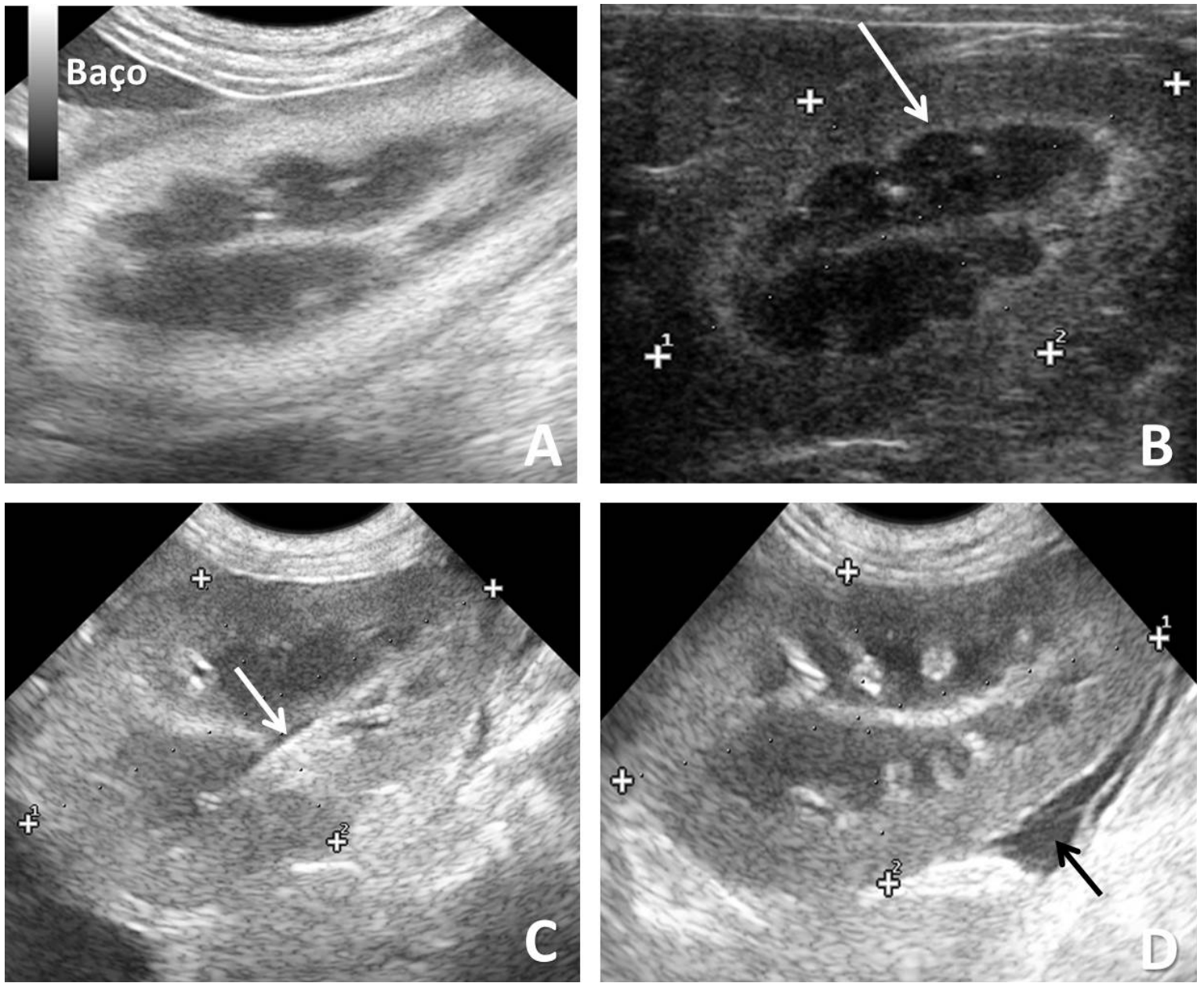

FIGURA 2 - Ultrassonografia dos rins de cães adultos com lesão renal tubular aguda: A aumento de ecogenicidade da cortical renal, hiperecoica em relação ao baço. B sinal de margem medular (seta). C - dilatação discreta da pelve renal (seta). D Efusão perirrenal (seta)

A pielectasia (Figura 2) pode ocorrer na pielonefrite aguda em cadelas com piometra (SANTOS et al., 2013), em decorrência de infecção ascendente do trato urinário inferior e em cães experimentalmente infectados (D'ANJOU et al., 2011). Dilatação pélvica renal discreta a moderada também tem sido observada em cães com leptospirose aguda (HOLLOWAY \& O'BRIEN, 2007; D'ANJOU et al., 2011; LUNN, 2011) e insuficiência renal aguda devido ao aumento da produção de urina na poliúria e obstrução parcial por debris celulares (MONTEIRO \& FROES, 2009). Embora a pelve renal não seja visualizada em rins normais, diurese salina também pode causar pielectasia moderada de até $3,0 \mathrm{~mm}$, às vezes unilateral, em cães (NYLAND \& MATTOON, 2005; D'ANJOU et al., 2011), portanto o uso de diurético ou pacientes submetidos a fluidoterapia devem ser considerados durante a avaliação.

Uma banda hiperecoica na junção corticomedular, conhecida como sinal de margem medular (Figura 2), pode ocorrer em casos de necrose tubular aguda devido à toxicidade por etilenoglicol e em nefrites infecciosas como na leptospirose e em animais com piometra (HOLLOWAY \& O'BRIEN, 2007; LANGSTON \& HEUTER, 2009; LUNN, 2011;). A presença de sinal de margem medular sugere uma anormalidade renal, porém esse aspecto pode ser encontrado com várias desordens e também foi observado em cães e gatos clinicamente normais. Por esse motivo, investigação mais completa é necessária para determinar a causa e o significado deste achado (NYLAND \& MATTOON, 2005). Efusão perirrenal (Figura 2) tem sido ENCICLOPÉDIA BIOSFERA, Centro Científico Conhecer - Goiânia, v.11 n.22; p.2101 2015 
reportada em cães com leptospirose ${ }^{21}$ e em nefrites infecciosas (HOLLOWAY \& OBRIEN, 2007; LUNN, 2011).

\section{ULTRASSONOGRAFIA EM MODO DOPPLER}

A ultrassonografia Doppler é utilizada para complementar a ultrassonografia em modo $\mathrm{B}$, uma vez que alterações ultrassonográficas da lesão renal aguda podem ser muito discretas, inespecíficas ou ausentes (IZUMI et al., 2000). O uso da ultrassonografia Doppler é útil para auxiliar no diagnóstico, uma vez que possibilita a avaliação da hemodinâmica renal que está diretamente ligada a seu funcionamento (CARVALHO, 2009).

Alterações da perfusão, em muitos casos, podem ser os primeiros sinais de que a funcionalidade renal está alterada. A diminuição da perfusão renal pode ocorrer por desordens glomerulares, tubulares e vasculares, causando muitas vezes alterações irreversíveis, que são assintomáticas em estágios iniciais. A possibilidade de antecipar uma possível piora do quadro clínico é muito importante para evitar lesões adicionais, que poderão levar a disfunção definitiva dos rins (MELO et al., 2006; CARVALHO, 2009).

O mapeamento com Doppler colorido permite determinar a presença ou ausência de fluxo no vaso, facilita a identificação das artérias e veias renais e possibilita a visualização das artérias e veias interlobulares e arqueadas que não são visualizadas em modo B (CARVALHO, 2009) (Figura 3).

Com o Doppler pulsado é possível identificar alterações na hemodinâmica por meio do traçado espectral, onde os dados sobre o desvio Doppler são representados na forma de gráficos, como um espectro temporal do sinal que retorna. O tempo decorrido fica no eixo horizontal ou linha de base e, a frequência de deslocamento Doppler pode ser vista no eixo vertical (CARVALHO, 2009). A detecção de desvio da frequência Doppler indica movimento do alvo, que na maioria dos casos está relacionado com a presença de fluxo. O sinal de desvio da frequência, positivo ou negativo, indica a direção do fluxo com relação ao transdutor (CARVALHO et al., 2008).
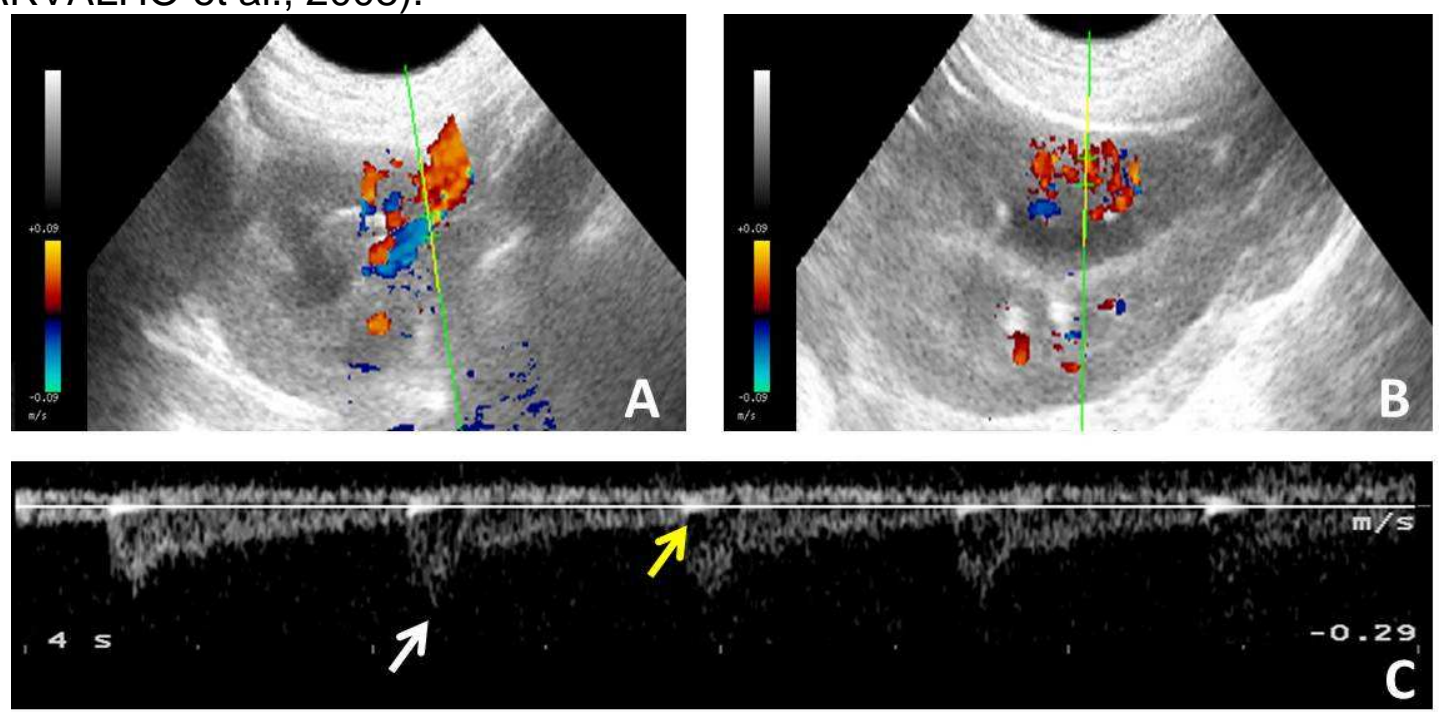

FIGURA 3 - Ultrassonografia Doppler colorido e pulsado do rim esquerdo de cão adulto. A Imagem em plano transversal na região do hilo renal evidenciando a artéria (azul) e a veia (vermelho) renal em corte longitudinal coloridas pelo Doppler. B - Imagem em plano sagital do rim evidenciando os vasos arqueados e interlobulares coloridos em azul e vermelho pelo Doppler. C - Traçado em Doppler pulsado do fluxo sanguíneo característico da artéria renal (seta branca - velocidade de pico sistólico, seta amarela - velocidade diastólica final) 
As artérias que suprem os rins, devido a estes requererem perfusão constante, apresentam fluxo contínuo, gradualmente decrescente no período diastólico e sem fluxo diastólico reverso (CARVALHO, 2009). O padrão é de baixa pulsabilidade e baixa resistividade de fluxo, com a presença de picos sistólicos amplos e contínuos e alta velocidade de fluxo na diástole com velocidade decrescente (Figura 3) (CARVALHO et al., 2008; CARVALHO, 2009; CARVALHO et al., 2009). A doença renal aguda pode alterar o padrão do fluxo sanguíneo de baixa resistividade da artéria renal para um padrão de alta resistividade (IZUMI et al., 2000), porém estas alterações no traçado podem ser muito discretas para serem detectadas visualmente (CARVALHO et al., 2008).

Para obter-se uma análise quantitativa do traçado Doppler, a maioria dos aparelhos tem a capacidade de calcular a média da frequência de deslocamento ou a velocidade automaticamente. O ponto máximo alcançado no espectro é denominado pico da velocidade sistólica (VPS) e o ponto mínimo na morfologia da onda é o valor da velocidade diastólica final (VDF) (Figura 3) (CARVALHO, 2009).

No entanto, informações sobre a impedância vascular não podem ser obtidas apenas pela velocidade absoluta, por isso foram desenvolvidos os índices Doppler (NOVELLAS et al., 2007). Esses índices comparam o fluxo durante a sístole e a diástole, sendo razões das velocidades obtidas do espectro Doppler. Por isso, ao contrário da análise isolada da velocidade, os índices têm como vantagem a independência da correção do ângulo, sendo usados para avaliação de vasos muito pequenos e tortuosos em que é difícil a correção do ângulo, como no caso das artérias intrarrenais (CARVALHO, 2009; BIGÉ et al., 2012).

Uma variedade de índices pode ser utilizada na avaliação renal com Doppler, destacam-se os índices de resistividade (IR) e pulsabilidade (IP), que fornecem informações acerca da resistência arterial ao fluxo sanguíneo, assim indicam alterações que resultam de uma variedade de enfermidades (NOVELLAS et al., 2007). Podendo ser utilizados para avaliar alterações nos fluxos dos órgãos que podem indicar doenças, para definir o prognóstico e para acompanhar o tratamento (IZUMl et al., 2000).

O IR, descrito por Pourcelot em 1974, relaciona o resultado da subtração entre as velocidades de pico sistólico e diastólico final sobre a velocidade de pico sistólico (IR = PVS - FVD/ PVS). O IP, descrito por Grosling e King em 1975, relaciona o resultado da subtração entre as velocidades de pico sistólico e diastólico final sobre a velocidade média (IP = PVS - FVD/ VM) (CARVALHO, 2009).

O aumento na velocidade diastólica leva a um correspondente aumento do fluxo sanguíneo e redução dos índices de resistividade e pulsabilidade. Portanto, baixa resistividade de fluxo sugere alto metabolismo do órgão ou tecido e altas resistências sugerem baixo metabolismo. Já índices de resistência aumentados reduzem o fluxo diastólico. Dependendo da alteração que está ocasionando o aumento da resistência, pode-se encontrar até ausência de fluxo diastólico ou a chamada diástole zero (CARVALHO, 2009).

A vantagem do IR é sua alta sensibilidade para diferenciar traçados anormais, porque o denominador nunca se torna zero, permitindo sempre a obtenção de um valor para este índice. O IP tem como vantagem o fato de levar em conta a velocidade média, que reflete o que ocorre durante todo o ciclo cardíaco, e não em apenas um momento específico como o IR (TORROJA, 2007).

O valor normal do IR para cães adultos saudáveis não sedados está entre 0,56 e 0,67 (MORROW et al., 1996; NOVELLAS et al., 2007; ASSIS, 2012) e do IP 
está entre 1,00 e 1,30 (NOVELLAS et al., 2007). De acordo com NOVELLAS et al. (2007) deve-se considerar como limite superior 0,72 para o IR e 1,52 para o IP, e RIVERS et al. (1997) sugerem que o limite superior do IR deve ser 0,70.

Em casos de necrose tubular aguda (NTA) há uma profunda redução do fluxo sanguíneo renal devido à intensa vasoconstrição intrarrenal, levando a uma resistência arterial elevada que pode ser vista mesmo no curso inicial da NTA, provocando mudanças nos índices Doppler. Estudos indicam que o IR é um importante marcador na IRA, visto que está elevado na maioria dos casos irreversíveis, sendo importante seu uso para o diagnóstico diferencial, prognóstico e acompanhamento da lesão renal aguda e IRA (MORROW et al., 1996; PLATT, 1997; RIVERS et al., 1997; IZUMI et al., 2000).

São as artérias interlobares e arqueadas que representam de forma mais fidedigna as alterações do córtex renal (CARVALHO, 2009) uma vez que a redução do fluxo sanguíneo renal ocorre principalmente devido a vasoconstrição intrarrenal, portanto o cálculo do IR e do IP é mais consistente nestas artérias (PLATT, 1997).

Estudo realizado por IZUMI et al. (2000) com seres humanos portadores de insuficiência renal aguda (IRA), apontou que o IR e o IP estavam aumentados em casos de necrose tubular aguda, mas não nos casos de azotemia pré-renal, revelando ausência de lesão no parênquima renal nestes casos. Assim, estes índices podem ser utilizados na diferenciação das causas de IRA. Neste estudo, a redução do IR e do IP foi relacionada à melhor prognóstico e melhora dos pacientes, enquanto que a elevação destes índices foi associada à pior prognóstico e falha em recuperar a função renal.

Na medicina veterinária estudos também mostram que os índices Doppler estão aumentados em cães e gatos com doença renal. SANTOS et al. (2013) observaram aumento do IR das artérias renais e intrarrenais em cadelas com piometra desidratadas devido a maior redução da perfusão renal, estando associados hipóxia, isquemia, injúria celular, apoptose celular e processos inflamatórios que cursam com necrose tubular aguda, presentes na injúria renal aguda. TORROJA (2007) observou IR e IP acima do limite superior em $38 \%$ e $43 \%$, respectivamente, dos cães com doença renal, e de ambos os índices em $73 \%$ dos gatos com IRC e 50\% dos gatos com IRA. RIVERS et al. (1997) observaram aumento do IR em $57,1 \%$ dos cães e em $90 \%$ dos gatos com doença renal, além de observarem retorno do IR para dentro dos valores de normalidade em dois cães e dois gatos com lesão renal aguda após a recuperação.

Portanto os índices Doppler são marcadores importantes na IRA, visto que estão elevados na maioria dos casos irreversíveis. Sendo importante seu uso para o diagnóstico, prognóstico e acompanhamento, uma vez que a redução dos valores está relacionada ao retorno da função renal (PLATT, 1997; RIVERS et al., 1997; IZUMI et al., 2000; SCHNELL \& DARMON, 2012).

MORROW et al. (1996) comparando os valores do IR com os achados histológicos de cães com doença renal, verificaram que todos os rins com IR acima de 0,70 tinham nefrite intersticial ou degeneração tubular, todos os rins com doença glomerular isolada apresentavam IR normais, demonstrando que os valores do IR estão mais relacionados a alterações túbulo intersticiais do que glomerulares. No entanto, valores acima do limite superior não são específicos para o tipo de doença renal, podendo ocorrer em doenças túbulo intersticiais (MORROW et al., 1996; RIVERS et al., 1997), NTA, neoplasias renais, cistos renais, hidronefrose (TORROJA, 2007) e na displasia renal congênita (MORROW et al., 1996). 
Embora esses índices sejam úteis, é importante ter em mente que essas medidas são influenciadas não apenas pela resistência ao fluxo nos vasos periféricos, mas por muitos outros fatores, inclusive a frequência cardíaca, a pressão sanguínea, o comprimento e a elasticidade dos vasos, bem como, a compressão orgânica extrínseca (CARVALHO, 2009). Doenças renais, hipotensão, redução da frequência ou do débito cardíaco, a presença de fluido subcapsular (PLATT, 1997), hepatopatias, diabetes e hiperadrenocorticismo podem elevar o valor do IR e do IP (TORROJA, 2007). Em humanos, algumas doenças como cardiopatia, hipertensão e diabetes também afetam os índices hemodinâmicos (PLATT, 1997; SCHNELL \& DARMON, 2012).

Além disso, o uso de alguns fármacos como sedativos, anestésicos e medicamentos cardiovasculares, podem atuar alterando sua resistência vascular (TORROJA, 2007; CARVALHO, 2009). Em estudo realizado por MELO et al. (2006) com 20 cães adultos sedados com $0,03 \mathrm{mg} / \mathrm{kg}$ de acepromazina via intramuscular, 10 minutos antes do exame, avaliou-se o IR das artérias renais e foram obtidos valores semelhantes aos encontrados na literatura para cães não sedados, demonstrando ser um protocolo que pode ser usado em animais não colaborativos e que não influencia nos valores do IR.

Estudos em humanos mostram que os índices podem variar de acordo com a idade, sendo encontrado IR mais elevado em crianças e idosos (PLATT, 1997). Em estudo realizado por ASSIS (2012) com cães não foi encontrada diferença significativa do IR e do IP entre os grupos de cães jovens (até 18 meses), adultos (19 meses a 10 anos) e idosos (mais de 10 anos) para a artéria renal, mas foi observada diferença entre o grupo entre cães adultos e idosos para as artérias arqueadas e interlobulares. Outro estudo em cães, realizado por MORROW et al. (1996), também não indicou diferença do IR entre as artérias renais de filhotes (menos de um ano) e de adultos (mais de um ano).

Considerando os inúmeros fatores que podem promover variação dos índices Doppler, a interpretação deve sempre considerar toda essa gama de variáveis (CARVALHO, 2009). No entanto, quando a elevação da resistividade arterial acompanha alterações na função renal, detectadas em exames laboratoriais, o IR e o IP podem estar relacionados a esta, podendo ser utilizados para avaliar a progressão ou regressão da doença renal (PLATT, 1997).

\section{CONSIDERAÇÕES FINAIS}

A lesão renal tubular aguda tem como principais causas a exposição a agentes nefrotóxicos e a isquemia renal, principalmente em pacientes de risco como cães idosos, com doença renal crônica, submetidos ao tratamento com medicamentos potencialmente nefrotóxicos e que diminuem o fluxo sanguíneo renal. O prognóstico é desfavorável e está associado à alta taxa de mortalidade nos cães que desenvolvem IRA, portanto o diagnóstico precoce é fundamental para garantir sucesso no tratamento.

Ultrassonografia em modo $B$ e em modo Doppler pulsado tem sido utilizada em associação com os exames laboratoriais para o diagnóstico, acompanhamento e determinação do prognóstico da lesão tubular aguda. No entanto na avaliação Doppler deve-se levar em consideração o uso de sedativos ou anestésicos, a presença de doenças concomitantes e a idade do paciente, pois estes fatores podem afetar o IR e o IP das artérias renais e intrarrenais. 


\section{REFERÊNCIAS}

ACIERMO, M.J.; LABATO, M.A. Hypertension in renal disease: diagnosis and treatment. Clinical Techniques in Small Animal Practice, Philadelphia; v. 20, p. 23-30, 2005.

ANDREADE, M.G. M. G.; LEME F.O.P.L.; PAES P.R.O.; GHELLER V.A. Avaliação da gama-glutamiltransferase na urina de cadelas com piometra. Vet. Not., Uberlândia; v. 20, n. 1, p. 1-7, 2014.

ARAÚJO, N. C.; RIOJA, L. S.; REBELO, M. A. P. A clinical predictor index for renal survival. Jornal Brasileiro de Nefrologia, São Paulo; v.32, n. 1, p. 27-32, 2010.

ASSIS, M. M. Q. Valores da dopplerfluxometria renal em cães clinicamente sadios de distintas faixas etárias. 2012. 67f. Dissertação (Mestrado) - Faculdade de Medicina Veterinária e Zootecnia, Universidade Estadual Paulista "Júlio de Mesquita Filho" Disponível em: <http://base.repositorio.unesp.br/bitstream/handle/>. Acesso em: 16 maio 2015

BAETGE, C.L.; MATTHEWS, N. S. Anesthesia and analgesia for geriatric veterinary patients. Veterinary Clinics of North America: Small Animal Practice, Philadelphia; v. 42, p. 643-653, 2012.

BARR, F. J.; HOLT, P. E.; GINNS, C. Ultrasonographic measurement of normal renal parameters. Journal Small Animal Practice, Oxford; v. 31, p. 180-184, 1990.

BERBEL, M.N.; PINTO M.P.R.; PONCE D.; BALBI A.L. Aspectos nutricionais na lesão renal aguda. Revista da Associação Médica Brasileira, São Paulo; v. 57, n. 5, p. 600-606, 2011.

BIGÉ, N.; LÉVY, P.P.; CALLARD, P.; FAINTUCH, J.M.; CHIGOT, V.; JOUSSELIN, V.; RONCO, P.; BOFFA, J.J.Renal arterial resistive index is associated with severe histological changes and poor renal outcome during chronic kidney disease. BMC Nephrology, London; v. 13, p. 139, 2012.

CARVALHO, A. M. P.; SALAVESSA, C. M.; SILVEIRA, L. S. Ultrassonografia e histopatologia renal em cães. Arquivo Brasileiro de Medicina Veterinária e Zootecnia, Belo Horizonte; v. 62, p. 105-1017, 2010.

CARVALHO, C. F. Ultrassom Doppler renal. In: em pequenos animais. São Paulo: Roca, p. 71-83. 2009.

Ultrassonografia Doppler

CARVALHO, C.F.; CERRI, G.G.; CHAMMAS, M.C. Parâmetros Doppler velocimétricos das artérias renais e da aorta abdominal em gatos da raça persa. Ciência Rural, Santa Maria; v. 39, n. 4, p. 1105-1110, 2009.

CARVALHO, C. F.; CHAMMAS, M. C.; CERRI, G. G. Morfologia duplex Doppler dos principais vasos sanguíneos abdominais em pequenos animais: revisão bibliográfica.

Ciência Rural, Santa Maria; v. 38, n. 3, p. 880-888, 2008. 
COLE, R. T.; MASOUMI A.; TRIPOSKIADIS F.; GIAMOUZIS G.; GEORGIOPOULOU V.; KALOGEROUPOULOS A.; BUTLER J. Renal dysfunction in heart failure. Medical Clinics of North America, Philadelphia; v.96, p. 955-974, 2012.

COOPER, R.L.; LABATO, M.A. Peritoneal dialysis in cats with acute kidney injury: 22 cases (2001-2006). Journal of Veterinary Internal Medicine, Lawrence; v. 25, p. 14-19, 2011.

COSTA, J.A.C.; VIEIRA NETO, O. M.; MOYSÉS NETO, M. Insuficiência renal aguda. Medicina, Ribeirão Preto; v. 36, p. 307-324, 2003.

CUNHA, L. M. F. Correlação entre peso corporal e volume renal por medidas lineares ultra-sonográficas em cães da raça Dachshund. 2005. 38f. Dissertação (Mestrado em Ciências Veterinárias) - Faculdade de Medicina Veterinária, Universidade Federal de Uberlândia, Uberlândia, 2005. Disponível em: < http://www.bdtd.ufu.br/tde_arquivos/6/TDE-2007-04-17T143443Z-

520/Publico/LMFCunhaDISSPRT.pdf>. Acesso em: 22 abr. 2015.

D'ANJOU, M.; BÉVARD, A; DUNN, M. E. Clinical significance of renal pelvic dilatation on ultrasound in dogs and cats. Veterinary Radiology and Ultrasound, Raleigh; v. 52, p. 88-94, 2011.

DIEHL, S. H., SESHADRI, R. Use of continuous renal replacement therapy for treatment of dogs and catswith acute or acute-on-chronic renal failure: 33 cases (2002-2006). Journal of Veterinary Emergency and Critical Care, San Antônio; v. 18 , n. 4 , p. 370-382. 2008.

DORVAL, P.; BOYSEN. S.R. Management of acute renal failure in cats using peritoneal dialysis: a retrospective study of six cases (2003-2007). Journal of Feline Medicine and Surgery, London; v. 11, p. 107-115, 2009.

FRANCEY, T.; SCHWEIGHAYSER, A. Epidemiologia clínica das doenças renais no gato. Veterinary Focus, London; v. 18, p. 23-30, 2009.

GRAUER, G. F. Early detection of renal damage and disease in dogs and cats. Veterinary Clinics of North America: Small Animal Practice, Philadelphia; v. 35, n. 3, p. 581-596, 2005.

HOLLOWAY, A.; O'BRIEN,R. Perirenal effusion in dogs and cats with acute renal failure. Veterinary Radiology and Ultrasound, Raleigh; v. 48, n. 6, p. 574-579, 2007.

INTERNATIONAL TENAL INTEREST SCIETY. Grading of acute kidney injury. Novart Anim Health, 2013. 7 p. Disponível em: < www.iris-kidney.com/IRIS GRADING OF ACUTE KIDNEY INJURY (final).pdf.>. Acesso em: 11 jul. 2015

IZUMI, M.; SUGIURA T.; NAKAMURA H.; NAGATOYA K.; IMAI E.; HORI M. Differential diagnosis of prerenal azotemia from acute tubular: necrosis and prediction of recovery diseases by Doppler ultrasound. American Journal of Kidney Disease, New York; v. 35, n. 4, p. 713-719, 2000. 
JOHN R.; HERZEMBER, A.M. Renal toxicity of therapeutic drugs. Journal of clinical pathology, London, v. 62, p. 505-515, 2009.

KHAN S. A.; MCLEAN, M. K. Toxicology of Frequently Encountered Nonsteroidal Anti-Inflammatory Drugs in Dogs and Cats. Veterinary Clinics of North America: Small Animal Practice, Philadelphia; v. 42, p. 289-306, 2012.

KUKANICH, B. Geriatric veterinary pharmacology. Veterinary Clinics of North America: Small Animal Practice, Philadelphia; v. 42, p. 631-642, 2012.

LANGSTON, C. E.; HEUTER, K. J. Leptospirosis a re-emerging zoonotic disease. Veterinary Clinics of North America: Small Animal Practice, Philadelphia, v. 33, p. 791-807, 2009.

LI, H.; LIU, Z.; LIU, X.; HAN, X.; KANG, H.Risk factors and outcome of acute renal failure in patients with severe acute pancreatitis. Journal of Critical Care, Orlando; v. 25 , p. 225-229, 2010.

LITTMAN M. P. Protein-losing nephropathy in small animals. Veterinary Clinics of North America: Small Animal Practice, Philadelphia; v. 41, p. 31-62, 2011.

LUNN, K. F. The kidney in critically ill small animals. Veterinary Clinics of North America: Small Animal Practic., Philadelphia; v. 41, n. 4, p. 727-744, 2011.

MELCHERT, A.; LAPOSY, C.B.; MOTTA, Y.P.; GARCIA, A.C.F.Z. Gama-glutamil transpeptidase urinária como indicador de insuficiência renal aguda induzida por gentamicina em cães. Arquivos de Ciências Veterinárias e Zoologia da Unipar, Umuarama,v. 10, n. 2, p. 111-116, 2007.

MELGAÇO, S.S.C.; SARAIVA, M.I.R.; LIMA, T.T.C.; SILVA JÚNIOR, G.B.; DAHER, E.F. Nefrotoxicidade dos anti-inflamatórios não asteroidais. Medicina, Ribeirão Preto; v. 43, n. 4, p. 382-900, 2010.

MELO, M. B.; VEADO, J.C.C.; SILVA, E.F.; MOREIRA, S.M.; PASSOS, L.M. F. Dopplerfluxometria das artérias renais: valores normais das velocidades sistólica e diastólica e do índice resistivo nas artérias renais principais. Arquivo Brasileiro de Medicina Veterinária e Zootecnia, Belo Horizonte; v. 58, n. 4, p. 691-693, 2006.

MENEZES, L.B.; FIORAVANTI, M.C.S.; SILVA, M.S.B.; FRANCO, L.G.; SALES P.T.; ANDRASCKO M.M.; VEADO J.C.C.; ARAÚJO E.G. Avaliação do efeito da clorpromazina sobre a função renal de cães submetidos à isquemia e reperfusão. Pesquisa Veterinária Brasileira, Rio de Janeiro; v. 30, n. 2, p. 108-114, 2010.

MONTEIRO, S. C.; FROES, T. R. Utilização da ultrassonografia no diagnóstico das nefromegalias em pequenos animais: revisão de literatura. Clínica Veterinária, São Paulo; v. 14, p. 54-58, 2009. 
MORROW, K.L.; SALMAN, M.D.; LAPPIN, M.R.; WRIGLEY, R. Comparison of the resistive index to clinical parameters in dogs with renal disease. Veterinary Radiology and Ultrasound, Raleigh; v. 37, n. 3, p. 193-199, 1996.

MYOTT M.; LANGSTON C. Differentiating between acute and chronic kidney disease. Veterinary Medicine, Chicago; p. 295-302. 2011.

NOVELLAS, R.; ESPADA, Y.; RUIZ DE GOPEGUI, R. Doppler ultrasonographic estimation of renal and ocular resistive and pulsatility indices in normal dogs and cats. Veterinary Radiology and Ultrasound, Raleigh; v. 48, n. 1, p. 69-73, 2007.

NYLAND, T.G.; MATTON J.S.; HERRSELL E.J.; WISNER E.R. Trato urinário. In: Nyland T G, Matton JS. Ultra-som diagnóstico em pequenos animais. 2 ed. São Paulo: Roca, p. 161-198.2005.

PALUMBO, M. I. P.; MACHADO, L. H. A.; ROMÃO, F. G. Manejo da insuficiência renal aguda em cães e gatos. Arquivos de Ciências Veterinárias e Zoologia da Unipar, Umuarama, v. 14, n. 1, p. 73-76, 2011.

PLATT, J. F. Doppler ultrasound of the kidney. Seminars in Ultrasound CT, and MRI, Philadelphia; v. 18, n. 1, p. 22-32, 1997.

POLZIN, D.J. Chronic kidney disease in small animals. Veterinary Clinics of North America: Small Animal Practice, Philadelphia, v. 41, n. 1, p. 15-30, 2011.

PRADO, T. D.; RIBEIRO, R. G.; BORGES, N. C.; NARDI, A. B. Aplicações e implicações da biópsia guiada por ultrassom em rim, fígado, baço e próstata de cães. Agrarian academy, Goiânia, v. 1, n. 1; p. 72-88, 2014.

RIVERS, B. J.; WALTER P.A.; POLZIN D.J.; KING V.L. Duplex doppler estimation of intrarenal pourcelot resistive index in dogs and cats with renal disease. Journal of Veterinary Internal Medicine, Philadelphia; v. 11, n. 4, p. 250-260, 1997.

ROSS E. A. Congestive Renal Failure: The Pathophysiology and Treatment of Renal Venous Hypertension. Journal of Cardiac Failure, Washington; v. 18, p. 930-938, 2012.

ROSS, L. Acute kidney injury in dogs and cats. Veterinary Clinics of North America: Small Animal Practice, Philadelphia; v. 41, n. 1, p. 1-14, 2011.

ROSS, S. Uremia aguda no gato. Veterinary Focus, London; v. 18, p. 23-30, 2009.

RUFATO, F. H.; REZENDE-LAGO, N. C. M; MARCHI, P.G. F. Insuficiência renal em cães e gatos. Revista Eletrônica da Univar, Barra do Garças; v.6, p. 167-173, 2011.

SAMPAIO, K. M. O. R, ARAÚJO, R. B. Ultra-sonografia de características lineares e estimativas do volume de rins de cães. Arquivo Brasileiro de Medicina Veterinária e Zootecnia, Belo Horizonte; v. 54, n. 3, p. 248-254, 2002. 
SANTOS, R.V. MERLINE, M.B.; SOUZA, L.P.; MACHADO, V.M.V.; PANTOJA, J.C.F.; PRESTES, N.C. Ultrassonografia Doppler na avaliação renal de cadelas diagnosticadas com piometra antes e após tratamento com ovariosalpingohisterectomia. Pesquisa Veterinária Brasileira, Rio de Janeiro; v. 33, n. 5, p. 635-642, 2013.

SCHNELL, D.; DARMON, M. Renal Doppler to assess renal perfusion in the critically ill: a reappraisal. Intensive Care Medicine, New York; v. 38, n. 11, p. 1751-1760, 2012.

TORROJA, R. N. Vascular resistance determination with Doppler ultrasound in canine and feline disease. 2007. 171f. Tese (Doutorado) - Facultat de Veterinària, Universitat Autònoma de Barcelona, Barcelona. Disponível em: http://www.tdx.cat/bitstream/handle/10803/5736/rnt1de1.pdf?sequence=1. Acesso em: 10 jan, 2015.

VADEN, S.L.; LEVINE, J., BREITSCHWERDT, E. B. A retrospective case-control of acute renal failure in 99 dogs. Journal of Veterinary Internal Medicine, Philadelphia; v. 11, n. 2, p. 58-64, 1997.

VEADO, J.C.C. Doença renal crônica. FVR discuss. Ed. esp., v. 19, 2011. 